\title{
Stochastic Models of Electromagnetic Vacuum in Resonance Phenomena
}

\author{
N.H. Cong ${ }^{1}$, T.D. Thanh ${ }^{2}$, T.N. Hoang ${ }^{3}$ \\ ${ }^{1}$ Electric Power University, Vietnam \\ ${ }^{2}$ Food Industry College of Ho Chi Minh City, Vietnam \\ ${ }^{3}$ Vinh University, Vietnam
}

(Received: 29 June 2010; accepted: 13 September 2010)

\begin{abstract}
In this article, we both presented the concept of coherent and squeezed stochastic vacuum and simultaneously specified the way to calculate resonant fluorescent spectrum in two- or three-level system with configuration $\Lambda$ in the presence of squeezed stochastic vacuum. The effects that occurred in the presence of squeezed stochastic vacuum were the change of both the width and the height of central spectrum and of additional narrow peaks. The impacts of squeezed vacuum on fluorescence spectrum represent the dependence on the squeezed parameter of both amplitude and phase. In the absence of unsqueezed vacuum, fluorescent spectrum returns to normal Mollow spectral type.
\end{abstract}

Key words: stochastic models, squeezed vacuum, Mollow spectrum

\section{INTRODUCTION}

As we all know, considering the stimulated light of the quantum system as a combination of light, i.e. a state of minimum unstability, fluorescence spectrum obtained by the transition in the quantum system has been studied in detail. However, in recent three to four decades, apart from the combined state, by experiments we have also discovered the compressed state of light fields. Since then, research on this issue has been vigorously conducted both in theory and in practice.

The successful creation of squeezed light has drawn attention of many physical researchers when researching the interaction of this type of light with atomic systems. Gardiner [1] has studied two-level atom in the presence of squeezed vacuum (consider squeezed vacuum as whitenoise) and point out that the reduction depends strongly on squeezed phase. Resonant fluorescence in the presence of squeezed vacuum has attracted attention and been concentratively researched. From the research results of resonant fluorescence in the presence of squeezed vacuum, Carmichael and colleagues [2] have showed that the line- width and sideband of resonant spectrum lines clearly depend on the squeezed phase. Two-level atomic spectrum becomes asymmetric.

A series of results related to the impact of squeeze on a two-level system has been mentioned in other works [3-6].

In previous theoretical researches, they generally used two-level approximation of the atomic samples. When taking notice of phase dependence of the combination, we found that the two-level approximation of the atomic system must be replaced by a three-level model. Atomic research which depends on the phase of squeezed vacuum will be further approximately asymptotic with a normal physical system in practice. The reason why a three-level atom in the electromagnetic field is less popular than the two-level one can so far be explained by the complexity of mathematical tools used in the studies.

Resonant fluorescence of a three-level atom in the presence of normal vacuum has been studied, for example in $[7,8]$. After a series of researches on the squeezed effects of two-level atom, physicists began studying interactions of three-level atom with squeezed light. Jagatap and colleagues [9] have studied resonant fluorescence of the 
three-level system with sigma configuration $\Xi$. Other kinds of configurations $\Lambda$ and $V$ have been studied in Smart and Swain's researches [10, 11].

It is well known that the starting point of researches on the interactions between atomic system and light in the presence of vacuum is to research damping as a result of vacuum fluctuation.

In this article, we will refer to the application of the stochastic descriptive method of vacuum in studying the resonant fluorescence of two- or three-level atoms. Specifically, we will mention the research on the capacity of resonant fluorescence spectrum (RFS) in the presence of coherence vacuum and squeezed vacuum.

\section{STOCHASTIC FLUCTUATION OF ELECTROMAGNETIC VACUUM}

As we all know, when studying the classic monochromatic light, the sine-shaped electric field $E(t)$ can perform as a sum of two variable complex quantity over time $a(t)$ and $a^{*}(t): E(t)=\left[a(t)+a^{*}(t)\right] / 2$. In the complex plane, they rotate contrariwise over time with $a(t)=a e^{-i \omega t} ; a^{*}(t)=a^{*} e^{i \omega t}$. Complex amplitude $a$ can be shown by $a=x+i p, \quad x, p$ here are real quantities. Then the strength of electric field is expressed by $x, p$ as follows:

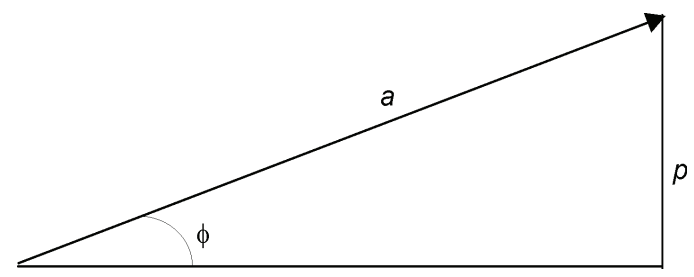

Fig. 1. Phase and perpendicular components represent the classical monochromatic field

$E(t)=x \cos \omega t+p \sin \omega t$ with $x=\left(a+a^{*}\right) / 2 ; p=\left(a+a^{*}\right) / 2 i$ As sine and cosine diphase $\pi / 2$, thus $x, p$ are perpendicular.

When switching to light quantum theory, we have:

$$
E(t) \rightarrow \hat{E}(t) ; a(t) \rightarrow \hat{a}(t) ; a^{*}(t) \rightarrow \hat{a}^{+}(t) ; x \rightarrow \hat{x} ; p \rightarrow \hat{p} .
$$

The laws of quantum mechanics define annihilation operator $\hat{a}(t)$ and creation operator $\hat{a}^{+}(t)$ as Hermitian operators, pursuant to the relation: $\left[\hat{a}(t), \hat{a}^{+}(t)\right]=1$ thus operators $\hat{x}, \hat{p}$ conform to the following communication relation: $[\hat{x}, \hat{p}]=i / 2$.

Therefore, according to the uncertainty principle, if we simultaneously measure two variables $x$ and $p$, then the error of measurement will follow Heisenberg's uncertainty relation.
The instability of the perpendicular components of quantum field (light field) in Descartes coordinates and polar coordinates which is illustrated in Fig. 2.

As a result, unlike classical electric field $E(t)$ which has a definite value at each time $t$, quantum electric field is always indefinite (there are many possible values of $E(t)$ at each time).

It is well known that the state of coherence has minimum uncertainty: $\sigma_{x} \sigma_{p}=1 / 4$.

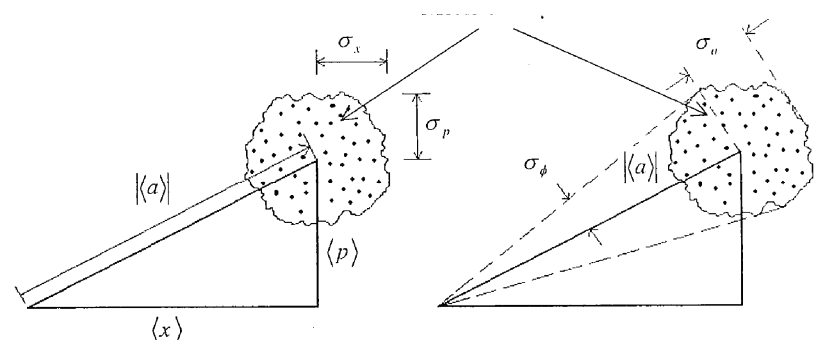

Fig. 2. Describe the uncertain region in the course of quantum mechanics on Descartes coordinates and polar coordinates

From the state of coherence, we can define a coherent vacuum state. It is the state where $\langle x\rangle=\langle p\rangle=0$, but still met the condition of $\sigma_{x} \sigma_{p}=\sigma_{n} \sigma_{\varphi}=1 / 4$. Besides, according to quantum mechanics, the energy on average of electromagnetic field is $\hbar \omega(\langle n\rangle+1 / 2)$. In the case of $\langle n\rangle=0$, the energy of field is $\hbar \omega / 2$ which represents the vacuum oscillation. Thus, the vacuum state of electromagnetic coherence is the vacuum state with deviations of perpendicular components $\sigma_{x}=\sigma_{p}=1 / 2$.

The vacuum state of time-dependent electric field can be illustrated in Fig. 3.
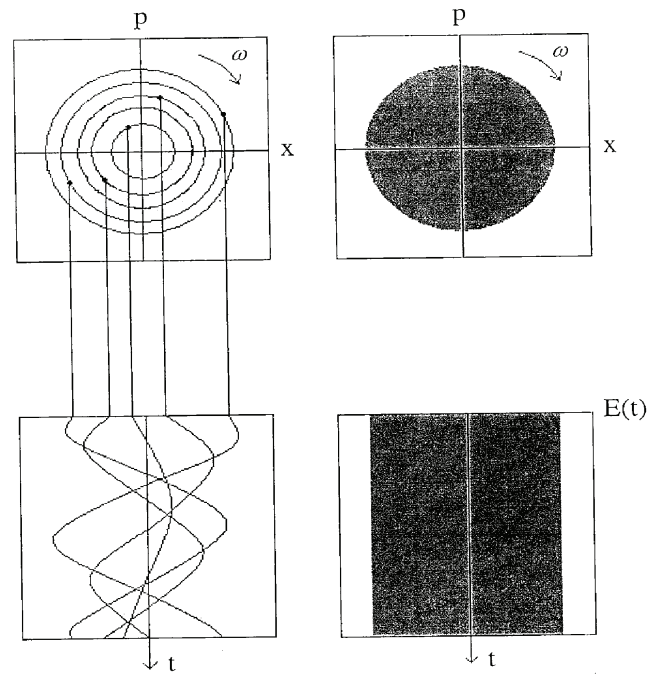

Fig. 3. Vacuum state of time-dependent electric field 
The vacuum state of time-dependent electric field can be illustrated in the figure on the left:

Apart from the concepts of coherent electric field and coherent vacuum, in the process of research on vacuum, we also mention the concepts of squeezed state and squeezed vacuum state.

They defined that a state is called perpendicular squeeze if one of the two perpendicular components has a deviation smaller than the value $1 / 2$ of the coherent state or coherent vacuum state. The perpendicular squeeze of light can be illustrated in the Fig. 4.

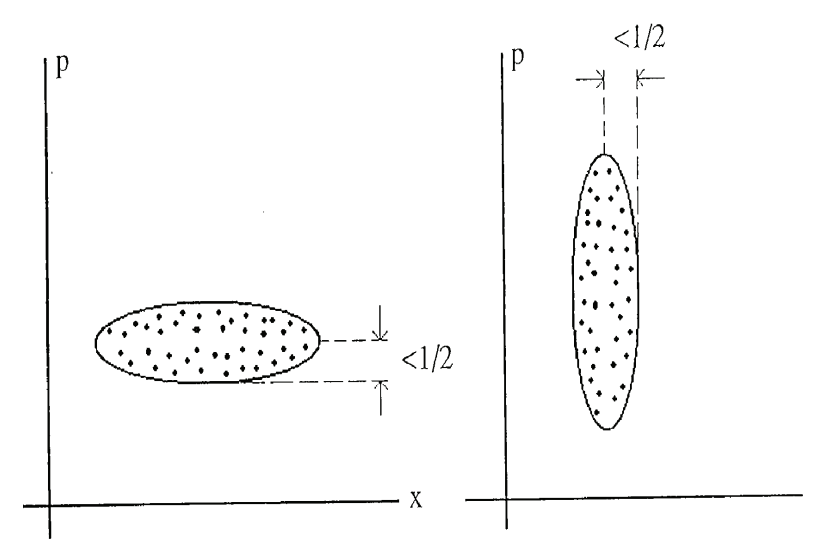

Fig. 4. Perpendicular squeezed state of light

Similarly, the squeezed vacuum state is defined as a vacuum state where an uncertainty of this perpendicular component is smaller than $1 / 2$ and the other of that perpendicular component is larger than $1 / 2$. That is if $\sigma_{x}<1 / 2$ then $\sigma_{p}>1 / 2$ and vice versa. Obviously, these uncertainties still have to satisfy $\sigma_{x} \sigma_{p} \geq 1 / 4$. When we meet both the above condition and the condition of $\sigma_{x} \sigma_{p}=1 / 4$, we will have the ideal state of squeezed vacuum. The squeezed vaccum state of electric field can be explained as follows:

With the aforementioned argument, we find that the oscillation of vacuum can totally be viewed as quantum interferences. The point is that, when examining the interaction of exciting field and quantum system, we should further consider these quantum interferences.

We should also note that interferences depend on the phase in squeezed state, but remain the same for all phases in unsqueezed state.

When examining interactions between electromagnetic field and quantum system, we consider the influence of vacuum as the influence of outer interferences. Furthermore, these interferences can be regarded as stochastic fluctuation. Therefore, coherent vacuum and squeezed, on the theoretical basis of random processes, are called coherent stochastic vacuum and squeezed stochastic vacuum.

\section{a) Coherent stochastic vacuum}

Based on the quantum theory, we find that electromagnetic vacuum is the cause for stochastic radiation process of quantum system [12]. If external fluctuations, which excite resonant radiative atoms, have a short time of coherence, we can demonstrate outer interferences by white noises with the average value and correlation function as follows:

$$
\begin{gathered}
\left\langle x_{k}(t)\right\rangle=0 \\
\left\langle x_{k}(t) x_{l}\left(t^{\prime}\right)\right\rangle=2 D_{k l} \delta\left(t-t^{\prime}\right) .
\end{gathered}
$$

When examining interactions between laser field and two-level atomic system in the presence of coherent vacuum fluctuation, we suppose the laser field includes coherent component $\Omega_{0}$ and incoherent additional complex component $\Omega_{t}=x_{t}+i y_{t}$ caused by thermal fluctuations. They are described by Gauss whit noises, with the correlation function among their real and virtual components is non-vanishing and in the following form:

$$
\begin{aligned}
& \left\langle x_{t}(t) x_{t}\left(t^{\prime}\right)\right\rangle=2 d_{x x} \delta\left(t-t^{\prime}\right), \\
& \left\langle y_{t}(t) y_{t}\left(t^{\prime}\right)\right\rangle=2 d_{y y} \delta\left(t-t^{\prime}\right) .
\end{aligned}
$$

Therefore, they define the diffusive matrix $d$ for the real and virtual components of white-noise thermal fluctuation as follows:

$$
d=\left[\begin{array}{cc}
d_{x x} & 0 \\
0 & d_{y y}
\end{array}\right]=\left[\begin{array}{ll}
\gamma & 0 \\
0 & \gamma
\end{array}\right] .
$$

Here, $\gamma$ is the natural damping rate of thermal fluctuation.

Coherent vacuum interference is demonstrated by the fluctuation of real and virtual components of vacuum Rabi amplitude:

$$
\Omega_{v}=x_{v}+i y_{v}
$$

with

$$
\begin{aligned}
& \left\langle x_{v}(t) x_{v}\left(t^{\prime}\right)\right\rangle=2 D_{x x} \delta\left(t-t^{\prime}\right), \\
& \left\langle y_{v}(t) y_{v}\left(t^{\prime}\right)\right\rangle=2 D_{y y} \delta\left(t-t^{\prime}\right) .
\end{aligned}
$$

Here, $D_{i i}(i=x, y)=A$ are components of diffusive matrix $D$ with $A$ as Einstein coefficient of stochastic radiation caused by interactions between dipole and vacuum fluctuation: 


$$
D=\left[\begin{array}{ll}
A & 0 \\
0 & A
\end{array}\right] .
$$

\section{b) Squeezed stochastic vacuum}

Usually we describe squeezed vacuum through two squeezed parameters $N, M$. These parameters are specific for quantum fluctuations of squeezed amplitude. The actual parameter $N$ related to the thermal distribution of squeezed vacuum, and parameter $M=|M| e^{i \varphi}$ demonstrates phasedependent vacuum distribution caused by squeezed quantum interferences. At that time the thermal component of squeezed vacuum has the same expression as in the case of coherent vacuum and its statistical properties are featured by diffusive matrix:

$$
d=A\left[\begin{array}{ll}
N & 0 \\
0 & N
\end{array}\right] .
$$

The phase-dependent components of squeezed vacuum interference can be demonstrated by diffusive matrix $D$ [2], [14]:

$$
D=\left[\begin{array}{cc}
1-2|M| \cos \varphi & 2|M| \sin \varphi \\
2|M| \sin \varphi & 1+2|M| \cos \varphi
\end{array}\right]
$$

This expression is entirely appropriate to the illustration shown in Fig. 5.

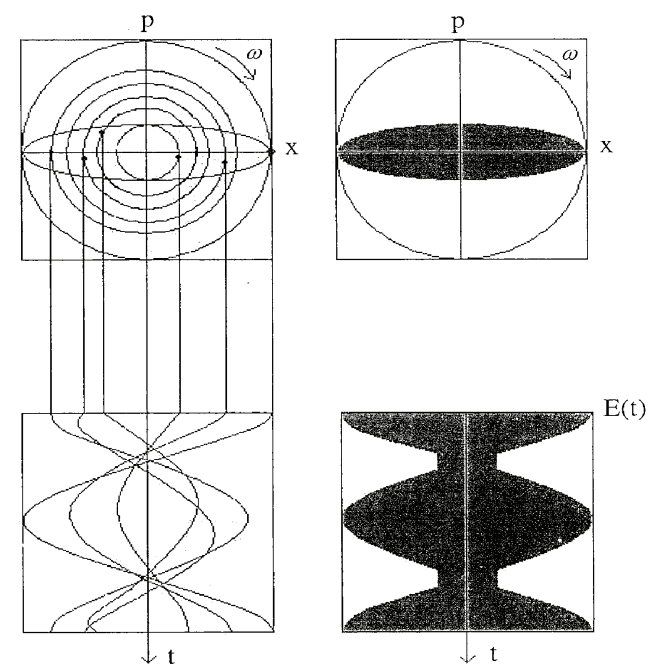

Fig. 5. Squeezed vacuum state of time-dependent electric field

In general case, these parameters satisfy the following inequality: $|M|^{2} \leq N(N+1)$.
The equal sign appears when perpendicular components of vacuum field meet the minimum indefinite conditions, then the vacuum is called ideal squeeze.

\section{RESONANT FLUORESCENT SPECTRUM IN THE PRESENCE OF STOCHASTIC VACUUM}

\section{For a two-level quantum system:}

To calculate the capacity of fluorescence corresponding to bipolar transition in the presence of quantum interference (interference of Rabi frequency in electromagnetic vacuum), we construct a random Bloch vector including four components $[6,12]$ :

$$
\begin{gathered}
V(t)=\left[V_{1}, V_{2}, V_{3}, V_{4}\right]= \\
=\left[\sigma_{21}(t+\tau) \sigma_{12}(t) ; \sigma_{12}(t+\tau) \sigma_{12}(t),\right. \\
\left.P_{21}(t+\tau) \sigma_{12}(t) ; \sigma_{12}(t)\right]
\end{gathered}
$$

with $P_{21}(t)=(w+1) / 2$.

We all know that the optical Bloch equation provides a quantum system in the presence of interference as follows:

$$
\frac{d\langle V(t)\rangle}{d t}=\left(-i M_{c}-\Sigma\right)\langle V(t)\rangle \text {. }
$$

a) In the case of coherent stochastic vacuum:

Using the results of [13] we can calculate the randomly decreased matrix $\Sigma$. Applying Laplace change to Bloch vector and formula for calculation of fluorescence spectrum, we can find the spectral type of resonance fluorescence in this case as follows (Figs. 6 and 7):
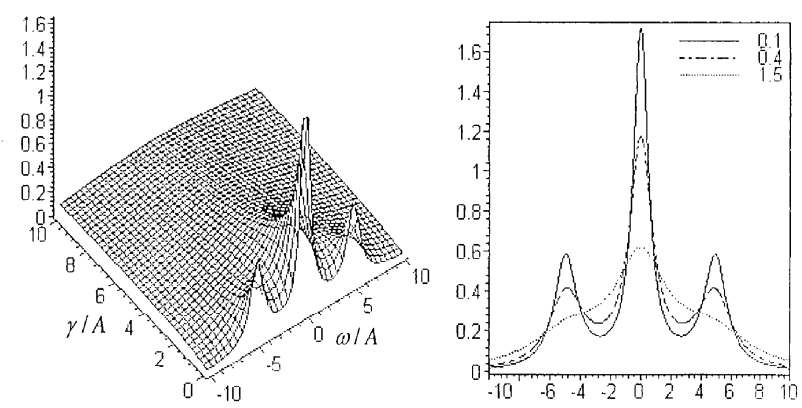

Fig. 6. The dependence of fluorescence spectrum on paramater $\gamma$

$$
\left(A=1 ; \Omega_{0}=5\right)
$$




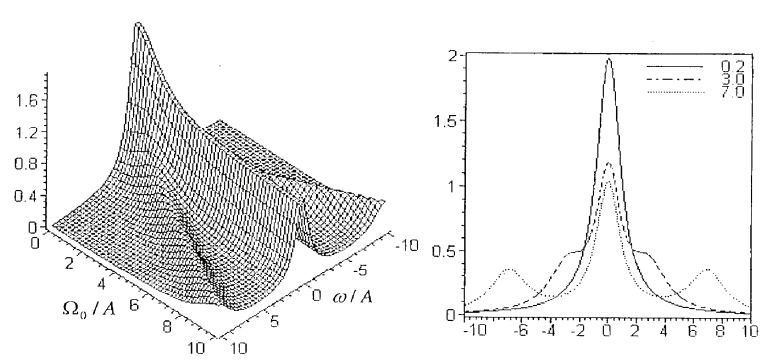

Fig. 7. The dependence of fluorescence spectrum on parameter $\Omega_{0} \quad(A=1 ; \gamma=0.5)$

\section{b) In the case of squeezed stochastic vacuum:}
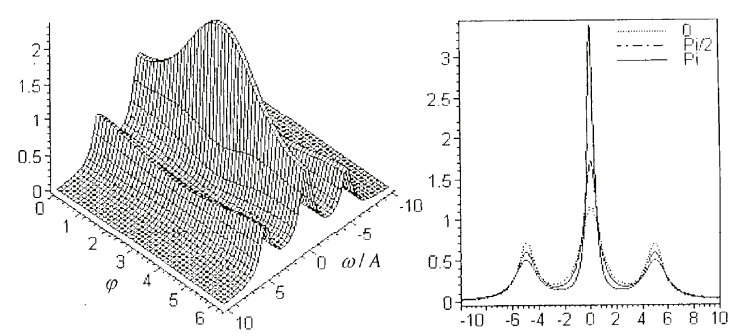

Fig. 8a. The dependence of fluorescence spectrum on squeezed phase $\varphi$, with $A=1 ; \Omega_{0}=5 ; N=0.1 ;|M|=0.3$

The dependence of fluorescence spectrum on descriptive spectral type in three-dimension is shown on the left of Fig. 8a, when the squeezed phase $\varphi$ varies in $0-2 \pi$. The form is common in the drawing two-dimension is shown on the right, with three values of $\varphi$ are $0, \pi / 2, \pi$. We found that when $\varphi$ varies in $0-\pi$, the height of main peak gradually increases and the height of two additional peaks gradually decreases.

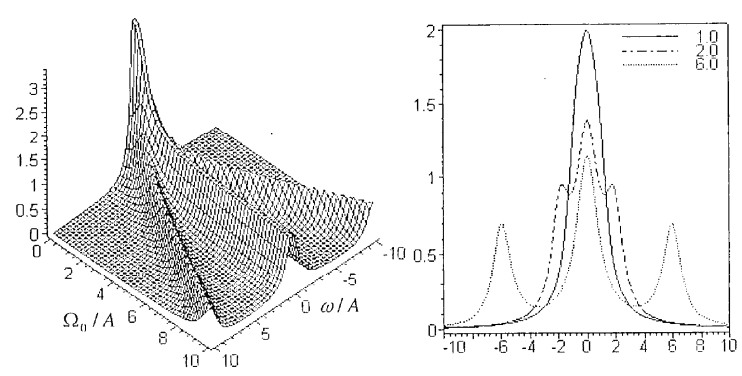

Fig. 8b. The dependence of fluorescence spectrum on frequency $\Omega_{0}$, with $A=1 ; \gamma=0.5 ; \quad N=0.1,|M|=0.3$

On the contrary, when $\varphi$ varies in $\pi-2 \pi$, the height of main peak gradually decreases while the height of two additional peaks gradually increases. During that period of change, the distance among peaks remains unchanged. It is noticeable that at the value of squeezed phase $\varphi=\pi$, the height of main peak reaches maximum value, which is appropriate to our prediction when studying the inversion of population: when there is a largest inversion of population between the two levels, spectral strength also reaches the maximum value.

The dependence of fluorescence spectrum on $\Omega_{0}$ in the presence of squeezed vacuum, with $A=1 ; \varphi=0 ; N=0.1$; $|M|=0.3$ is presented in Fig. $8 \mathrm{~b}$.

The spectral type in three-dimension is shown on the left, when parameter $\Omega_{0} / A$ varies in 1-10. The spectral type in two-dimension is shown on the right, with three peak gradually increases and the height of two values of $\Omega_{0} / A$ are 1.0, 2.0, and 6.0. From the spectral shape illustrated in two- and three-dimension, we find that, when $\Omega_{0}$ gradually increases from 0 , from only one peak in the beginning, two additional peaks will appear step by step with gradual reduction of all three in height. At the same time, the rate of height and the distance among peaks will change as the strength of outer field increases. This shape is fairly suitable to the experimental spectrum [14]

The dependence of fluorescence spectrum on squeezed parameters $N, M$ with $A=1 ; \varphi=\pi / 2 ;|M|^{2}=N(N+1)$ is presented in Fig. 9.

The spectral type in three-dimension is shown on the left, when squeezed parameter $N$ varies in $0-5$. The spectral type in two-dimension is shown on the right, with three values of $N$ are $0,1.0$, and 4.0. From the spectral shape illustrated in two- and three-dimension, we find that, when $N, M$ gradually decreases to 0 , the spectral graph clearly has three peaks; and when $N, M$ increase, two additional peaks will be gradually annulled and the height of the central peak will also change. Obviously, we also find that when $N=M=0$ (no longer affected by squeezed vacuum), it then coincides with the graph in case of coherent vacuum. It is also the wellknown Mollow spectrum itself.

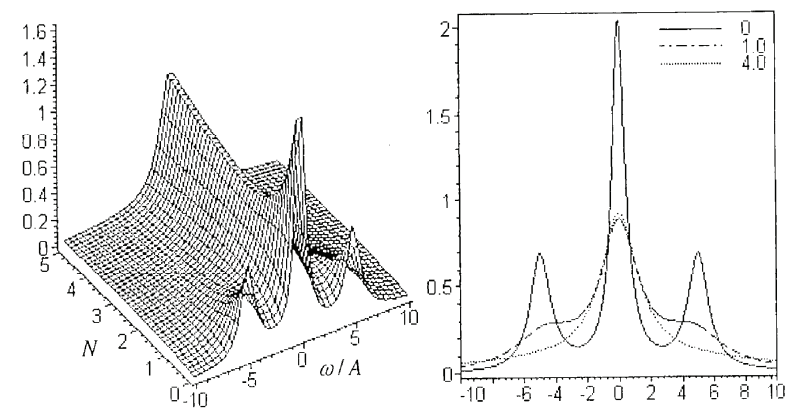

Fig. 9. The dependence of fluorescence spectrum on squeezed parameters $N, M$, with $A=1 ; \varphi=\pi / 2 ;|M|^{2}=N(N+1)$ 


\section{For the three-level quantum system:}

We consider a closed, $\Lambda$-type three-level system with two near-degenerate levels $|1\rangle ;|2\rangle$, and an excited as shown in Fig. 10.

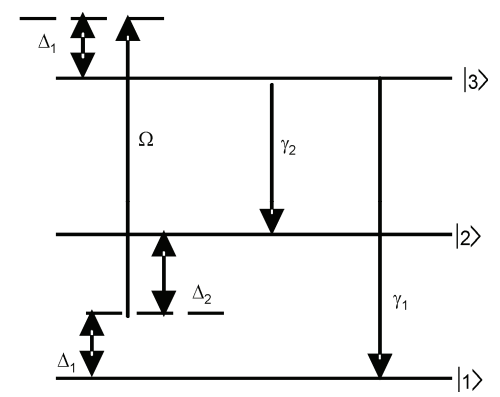

Fig. 10. Schema of three-level system

In order to take into account the induced-coherence effects by spontaneous emission, the upper level $|3\rangle$ is coupled to lower levels $|1\rangle ;|2\rangle$ by the same vacuum modes. The resonant frequencies between the upper level $|3\rangle$ and the ground levels are $\omega_{31}, \omega_{32}$, respectively. Note that: $\omega_{31}-\omega_{32}=\omega_{21}$ being the frequency separation of the lower levels. Our aim is to study interference effects and fluorescence spectrums in the given system. Therefore, we assume the two lower levels are closed-spaced with $\Delta_{1}+\Delta_{2}<\gamma_{1}, \gamma_{2}$ and $\Delta_{1}+\Delta_{2}=\omega_{31}-\omega_{32}$. Both transitions $|3\rangle \rightarrow|2\rangle$ and $|3\rangle \rightarrow|1\rangle$ are driven by laser field of frequency $\omega_{L}$ with the respective Rabi frequencies $\Omega_{1}$ and $\Omega_{2} . \quad \Delta_{3}=\omega_{L}-\omega_{32}-\Delta_{1}$ is the common detuning of the driving laser field.

The Hamiltonian of the system in the rotating wave approximation is given by [15]:

$$
\begin{aligned}
H= & \hbar \sum_{n=1}^{3} \omega_{n}|n\rangle\left\langle n\left|+\hbar \sum_{k \lambda} \omega_{k \lambda} a_{k \lambda}^{+} a_{k \lambda}-\hbar \sum_{n=1}^{2} g_{n k} a_{k \lambda}\right| 3\right\rangle\langle n|+ \\
& +\hbar \Omega_{1} e^{-i\left(\omega_{L} t+\varphi\right)}|3\rangle\left\langle 2\left|+\hbar \Omega_{2} e^{-i\left(\omega_{L} t+\varphi\right)}\right| 3\right\rangle\langle 1|+H . c .
\end{aligned}
$$

where $\hbar \omega_{n}$ are the energies of the atomic levels, and $a_{k \lambda}\left(a_{k \lambda}^{+}\right)$is the anihilation (creation) operator of the $k$-th mode of the vacuumfield with polarization $\vec{e}_{k \lambda}(\lambda=1.2)$ and angular frequency $\omega_{k \lambda}$. The parameter $g_{n k}$ is the coupling constant of the atomic transition $|n\rangle \rightarrow|3\rangle$ with the vacuum electromagnetic mode:

$$
g_{n k}=\sqrt{\frac{\omega_{k \lambda}}{2 \hbar \varepsilon_{0} V}}\left(\vec{\mu}_{3 n} \vec{e}_{k \lambda}\right)
$$

where $\vec{\mu}_{3 n}$ is the dipole moment of the transition $|n\rangle \rightarrow|3\rangle$.

We now assume that the quantized radiation field is in a broadband squeezed vacuum state with carrier frequecy $\omega_{v}$, wich is tuned close to the frequency of the atomic transitions $|3\rangle \rightarrow|2\rangle$ and $|3\rangle \rightarrow|1\rangle$, that is, $2 \omega_{v}=\omega_{32}+\omega_{31}$. The bandwidth of the squeezed field is assumed to be broad enough so that the squeezed vacuum appears as $\delta$ - correlated squeezed white noise to the atom. The correlation function for the field operators $a\left(\omega_{k \lambda}\right)$ and $a^{+}\left(\omega_{k \lambda}\right)$ can be written as [15]:

$$
\begin{gathered}
\left\langle a\left(\omega_{k \lambda}\right) a^{+}\left(\omega_{k \lambda}^{\prime}\right)\right\rangle=\left[N\left(\omega_{k \lambda}\right)+1\right] \delta\left(\omega_{k \lambda}-\omega_{k \lambda}^{\prime}\right), \\
\left\langle a^{+}\left(\omega_{k \lambda}\right) a\left(\omega_{k \lambda}^{\prime}\right)\right\rangle=N\left(\omega_{k \lambda}\right) \delta\left(\omega_{k \lambda}-\omega_{k \lambda}^{\prime}\right), \\
\left\langle a\left(\omega_{k \lambda}\right) a\left(\omega_{k \lambda}^{\prime}\right)\right\rangle=M\left(\omega_{k \lambda}\right) \delta\left(2 \omega_{v}-\omega_{k \lambda}-\omega_{k \lambda}^{\prime}\right),
\end{gathered}
$$

where $N\left(\omega_{k \lambda}\right)$ and $M\left(\omega_{k \lambda}\right)$ being slowly varying functions of the frequency that characterize the squeezing. Note that $M$ is a complex magnitude so that $M\left(\omega_{k \lambda}\right)=\left|M\left(\omega_{k \lambda}\right)\right| e^{i \varphi_{v}}$, where $\varphi_{v}$ is the phase of squeezed vacuum.

Through an appropriate unitary transformation we eliminate the explicit temoral dependence in the master equation, and obtain the evolution Bloch equations for the following density-matrix elements

$$
\begin{gathered}
V(t)=\left[V_{1}, V_{2}, V_{3}, V_{4}, V_{5}, V_{6}, V_{7}, V_{8}\right]= \\
=\left[\left\langle\sigma_{33}(t)\right\rangle,\left\langle\sigma_{33}(t)\right\rangle,\left\langle\sigma_{32}(t)\right\rangle,\left\langle\sigma_{23}(t)\right\rangle,\right. \\
\left.\left\langle\sigma_{31}(t)\right\rangle,\left\langle\sigma_{13}(t)\right\rangle,\left\langle\sigma_{21}(t)\right\rangle,\left\langle\sigma_{12}(t)\right\rangle\right] .
\end{gathered}
$$

Using calculation methods for fluorescence spectrum (see for example $[5,12]$ ), after some tedious but simple algebraic calculations, we find out fluorescence spectrum in the presence squeezed vacuum.

It is well known that this spectrum is proportional to the Fourier transformation of the steady-state correlation function: $\lim \left\langle E^{+}(t+\tau) E^{-}(\tau)\right\rangle$, where $E^{ \pm}(r, t)$ is the positive/negative frequency part of the radiation field in the far zone. The resonance fluorescence spectrum (RFS) can be expressed in terms of atomic correlation function:

$$
S(\omega)=\operatorname{Re}\left[\lim _{t \rightarrow \infty} \int_{0}^{\infty}\left\langle D^{+}(t+\tau) D^{-}(\tau)\right\rangle e^{-i \omega \tau} d \tau\right]
$$

where Re denotes the real part and $D^{ \pm}(t)$ is the atomic polarization operator: 


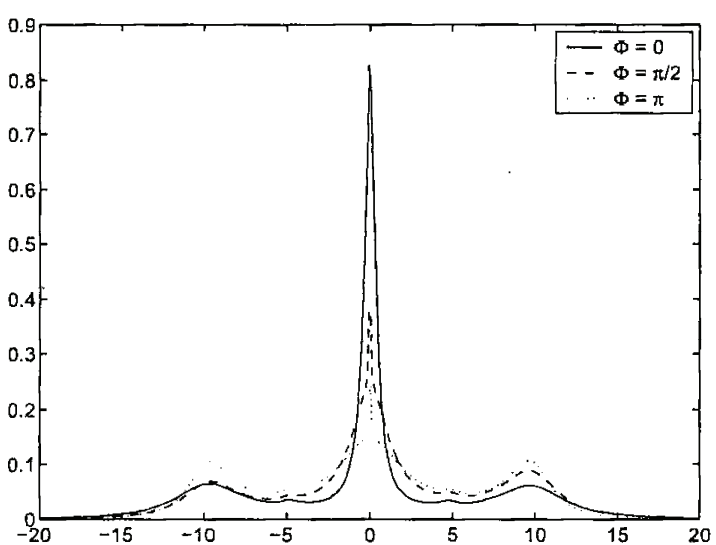

Fig. 11. The incoherent resonance fluorescence spectrum

$$
\begin{gathered}
\text { as a function of }\left(\omega-\omega_{L}\right) \\
\Delta_{1}=\Delta_{2}=0.1, \Delta_{3}=0, p=1, N=0.2, p=1, \\
\Omega_{1}=3, \Omega_{2}=4, \gamma_{1}=\gamma_{2}=\gamma=1 \\
D^{+}(t)=\vec{\mu}_{32} \sigma_{32}(t)+\vec{\mu}_{31} \sigma_{31}(t), \\
D^{-}(t)=\vec{\mu}_{32} \sigma_{23}(t)+\vec{\mu}_{31} \sigma_{13}(t) .
\end{gathered}
$$

In the following analysis, we are only interested in the incoherent part of RFS, which is given by:

$$
S_{\text {inc }}(\omega)=\operatorname{Re}\left[\lim _{t \rightarrow \infty} \int_{0}^{\infty}\left\langle\Delta D^{+}(t+\tau) \Delta D^{-}(\tau)\right\rangle e^{-i \omega \tau} d \tau\right],
$$

where $\Delta D^{ \pm}(\tau)=D^{ \pm}(\tau)-\left\langle\Delta D^{ \pm}(\infty)\right\rangle$ stands for the deviation of the dipole polarization operator from its mean steady-state value.

We present in Figs. 11 and 12 some acquired fluorescence spectra for three-level system in the presence squeezed vacuum [16]:

We have note that, when $N$ increaes, the height of the central peak decreases while the sidebands spread. The outer sidebands are modified in height and width by the squeezed vacuum. The effect of the squeezed phase on the shape of the spectra is clearly revealed in the case shows in Fig. 11. The spectrum displays a five-peak structure: the inner sidebands become appreciable as the splitting increases.

The central line and outer sidebands are highly modified by squeezed phase. The spectral asymmetry is clearly shown when $\varphi=\pi / 2$. Figure 12 shows that two inner peaks distinctly increase along with the augmentation of squeezed parameters and we can see that height of the peaks of spectra is modified by squeezed vacuum.

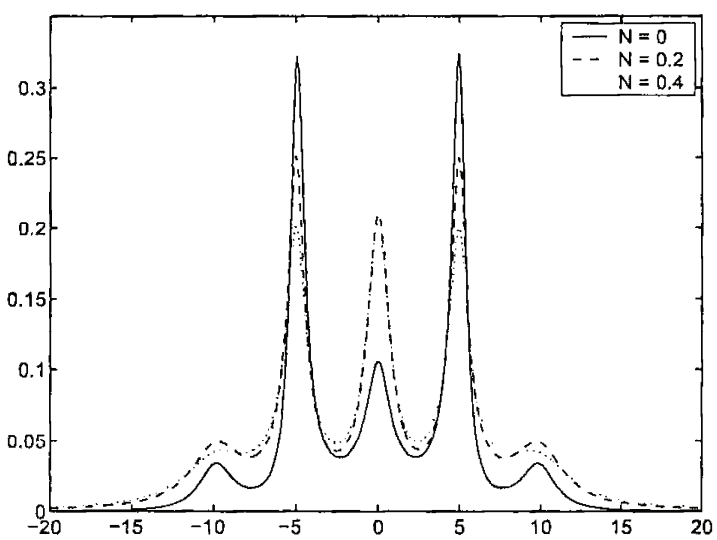

Fig. 12. The incoherent resonance fluorescence spectrum as a function of $\left(\omega-\omega_{L}\right)$ $\Delta_{1}=\Delta_{2}=0.1, \Delta_{3}=0, p=0, \Phi=0$, $\Omega_{1}=3, \Omega_{2}=4, \gamma_{1}=\gamma_{2}=\gamma=1$

\section{CONCLUSION}

In this article we both presented the concept of coherent stochastic vacuum and squeezed stochastic vacuum and simultaneously specified the way to calculate resonant fluorescent spectrum in two- or three-level system with configuration $\Lambda$ in the presence of squeezed stochastic vacuum. The effects occurred in the presence of squeezed stochastic vacuum is the change of both the width and the height of central spectrum and of additional narrow peaks. The impacts of squeezed vacuum on fluorescence spectrum represent the dependence on the squeezed parameter of both amplitude and phase. In the absence of unsqueezed vacuum, fluorescent spectrum returns to normal Mollow spectral type.

\section{References}

[1] C.W. Gardiner, Phys. Rev. Lett. 56, 1917 (1986).

[2] H.J. Carmichael et al., J. Mod. Optics 34, 821 (1987).

[3] A.S. Parkins, P.L. Gardiner, Phys. Rev. A 37, 3867 (1988).

[4] G.M. Palma, P.L. Knight, Optics Commun. 73, 131 (1989).

[5] K. Wódkiewicz, N.H. Cong, Optical resonance equations in the presence of strong radiation and short-noise collisions. Procceding of the International conference on Coherence and Quantum Optics (New York) (1990).

[6] N.H. Cong, L.V. Vinh, Collisional line shapes in the presence of broaband squeezed vacuum. Acta Phisyca Polonica (Poland) (2001).

[7] L.M. Narducii et al., Phys. Rev. A 42, 3867 (1990)

[8] A.S. Manka et al., Phys. Rev. A 43, 1630 (1990).

[9] B. N. Jagatap et al., Phys. Rev. A 43, 535 (1991).

[10] S. Smart, S. Swain, J. Mod. Optics 40, 1939 (1993). 
[11] S. Smart, S. Swain, J. Mod. Optics 41, 1055 (1994).

[12] E. Kahle, K. Wódkiewicz, J. Mod. Optics 41, 3, 491 (1994).

[13] T.N. Hoang, Graduate Thesis, Vinh University, Vietnam 47-49, 54-55 (2004).

[14] R.D. DeVoe, R.G. Brewer, Phys. Rev. Lett. 50, 17, 1296 (1983).
[15] M.R. Ferguson, Z. Fice, B.J. Dalton, Phys. Rev. A 54, 2379 (1996).

[16] T.D. Thanh, L.V. Nam, N.H. Cong, The 34th National Conference on Theoretical Physics (Vietnam) (3-6/8/2009).

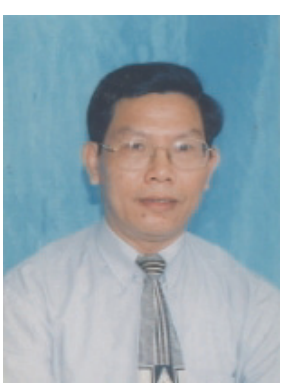

NguYen Huy CONG was born in Ha Tinh, Vietnam in 1947. He completed a B.Sc. degree in Physics at Hanoi University, Vietnam in 1969 and later went on to complete a $\mathrm{PhD}$ degree program in Physics at Warsaw University, Poland in 1988. His PhD dissertation entitled "Effective Bloch Equations in strong laser field" was supervised by Prof. K. Wódkiewicz. Research interests of Prof. N.H. Cong include fundamental aspects of quantum optics, particularly multi-photon processes, statistical properties of light, stochastic methods and chaos, and atomic coherence. He has worked as a lecturer since 1969, and then as an Associate Professor since 2002 for the Faculty of Physics at Vinh University, Vietnam. He is now the Director of Scientific Management and International Cooperation department at Electric Power University, Vietnam.

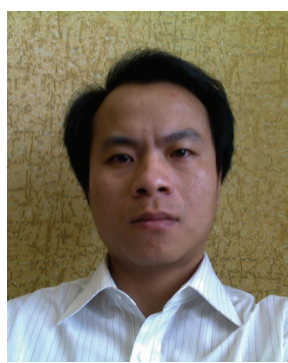

Trinh Ngoc Hoang was born in Thanh Hoa province, Vietnam. He graduated in physics from Vinh University in 2002. He completed his MSc study in Vinh University in 2004 under the supervision of professor Nguyen Huy Cong. Presently, he completes his PhD dissertation at Belarusian State University, The Republic of Belarus Institute of Physics, under the supervision of Prof. Zazhogin Anatoly Pavlovich. He is interested in Spectroscopy and laser. 